\title{
Which Friction Factor Model Is the Best? A Comparative Analysis of Model Selection Criteria
}

\author{
Ahmed H. Kamel ${ }^{1}$, Ali S. Shaqlaih ${ }^{2}$ and Arslan Rozyyev ${ }^{1}$ \\ 1. University of Texas of the Permian Basin Odessa, Texas 79762, USA \\ 2. University of North Texas at Dallas, Dallas, Texas 75241, USA
}

\begin{abstract}
The ongoing research for model choice and selection has generated a plethora of approaches. With such a wealth of methods, it can be difficult for a researcher to know what model selection approach is the proper way to proceed to select the appropriate model for prediction. The authors present an evaluation of various model selection criteria from decision-theoretic perspective using experimental data to define and recommend a criterion to select the best model. In this analysis, six of the most common selection criteria, nineteen friction factor correlations, and eight sets of experimental data are employed. The results show that while the use of the traditional correlation coefficient, $\mathrm{R}^{2}$ is inappropriate, root mean square error, RMSE can be used to rank models, but does not give much insight on their accuracy. Other criteria such as correlation ratio, mean absolute error, and standard deviation are also evaluated. The AIC (Akaike Information Criterion) has shown its superiority to other selection criteria. The authors propose AIC as an alternative to use when fitting experimental data or evaluating existing correlations. Indeed, the AIC method is an information theory based, theoretically sound and stable. The paper presents a detailed discussion of the model selection criteria, their pros and cons, and how they can be utilized to allow proper comparison of different models for the best model to be inferred based on sound mathematical theory. In conclusion, model selection is an interesting problem and an innovative strategy to help alleviate similar challenges faced by the professionals in the oil and gas industry is introduced.
\end{abstract}

Key words: Friction factor, information theory, model selection, turbulent flow, straight tubing.

\section{Nomenclature}

$\begin{array}{ll}\text { MARE } & \text { Mean absolute relative error, \% } \\ R M S E & \text { Root mean square error, \% } \\ S D_{\text {MARE }} & \text { Standard deviation of MARE } \\ C R & \text { Correlation ratio } \\ A I C & \text { Akaike Information Criterion } \\ f & \text { Friction factor, dimensionless } \\ g & \text { Ratio of evidence of a model } \\ i & \text { Any models in the set } \\ m & \text { Number of candidate models } \\ \underline{n} & \text { Flow behavior index, dimensionless } \\ N & \text { No. of data points } \\ N_{R e g} & \text { Generalized Reynolds number, dimensionless } \\ R^{2} & \text { Coefficient of determination } \\ K & \text { Number of parameters in the model } \\ y_{i} & \text { Actual value of the observed parameter } \\ \hat{y}_{i} & \text { Estimated value of the observed parameter } \\ \bar{y}_{i} & \text { Average value of the observed parameter }\end{array}$

Corresponding author: Ahmed Kamel, associate professor, research field: petroleum engineering. E-mail: kamel_a@utpb.edu.

\author{
w Akaike's weight \\ $\Delta \quad$ AIC difference, $\Delta_{\mathrm{i}}=\mathrm{AIC}_{\mathrm{i}}-\mathrm{AIC}_{\mathrm{min}}$
}

\section{Introduction}

Crude oil and other petroleum products are transported under turbulent flow conditions through pipelines. Pumping stations provide power to overcome the inertia, gravity, and friction inside the pipeline, where friction is the major component. For friction pressure losses calculations, the friction factor has to be determined first. This a crucial task partly due to the large number of correlations available to predict the friction factor for non-Newtonian pseudoplastic shear-thinning fluids. In many scenarios, these models are either simple but not accurate or are accurate but not simple. Yet, they all seem to suffer from some drawbacks. Generally, these models were not developed using independent data. They were either developed for a specific type of material and/or 
based on a particular set of results. Consequently, they are not universally applicable [1].

The other concern is due to the lack of complete fundamental understanding for turbulent phenomenon. There have been no general theories and/or mathematical and computational models to describe turbulent flow [2]. Instead, different analytical, semi-empirical and empirical correlations (explicit and implicit) have been proposed to predict friction factor under turbulent flow conditions [1].

Traditionally, the coefficient of determination, $R^{2}$ has been the criterion to select the best model; the model with the highest $R^{2}$ should be the one to be picked. Nowadays, statisticians are involved heavily in the question of model selection and there have been many approaches proposed over the years for dealing with this key issue. However, it is well documented that these approaches still have some shortcomings [3-5].

Considering this, the question to be answered is which model selection criteria should be adopted to pick the most accurate model among all models used to predict specific parameter. The very least we need is an approach that can be carried out easily and yields results that can be scientifically and numerically interpreted. In addition, it is important that the choice of model should be based on its general applicability, not just goodness-of-fit [6].

In this paper, a comprehensive and critical review of the various model selection criteria is presented. A total of six different criteria are evaluated using nineteen friction factor correlations and eight different sets of experimental data covering a wide range of flow behavior indices, $n$ and generalized Reynolds numbers, $N_{R e g}$. The first objective is to recommend a simple, but an accurate model selection criterion that can be unquestionably adopted by the professionals. The second objective is to apply the selected criterion to evaluate the available friction factor correlations and check their validity to recommend the most applicable one.

\section{Friction Factor Models}

The matrix involved in this study includes correlations that are used to calculate friction factor for non-Newtonian pseudoplastic fluids flowing in circular pipes under turbulent flow conditions. There are many correlations available in the literature to determine friction factor. Yet, they are not considered in this study due to either complexity or limited application range. Among all the available models, only nineteen models are used in the present study. These models were selected due to their higher accuracy and precision, simplicity, and their wide application range. The flow behavior index, $n$ ranges from 0.14 to 1.0 while the generalized Reynolds number ranges from 548 to 250,000 [1]. These models are listed in Table 1. More details can be found elsewhere $[1,7,8]$.

\section{Experimental Data}

The experimental data include eight different sets of friction factor data points measured at different values of flow behavior indices and generalized Reynolds numbers gathered by several authors. Dodge and Metzner [9] published friction factor values for flow behavior indices of $0.617,0.726$ and 1.0 while Shaver and Merrill [10] published friction factor values for flow behavior indices of $0.6,0.7$ and 0.9. Yoo [11] gathered friction factor for flow behavior indices covering a wide range from 0.241 to 0.893 while Szilas et al. [12] data were gathered for $n=0.5287$, 0.6991, 0.7169, 0.8311, and 0.948. Bogue [13] published another set of friction factor data covering a wide range of flow behavior indices ranging from 0.445 to 1.0 . Other sets of data were gathered by Pinho and Whitelaw [14], Pereira and Pinho [15], and Cruz and Pinho [16] covering an $n$ range from 0.4 to 0.9. The included data sets cover a wide range of generalized Reynolds number up to 100,000 . The details of the experimental data, ranges for flow behavior indices, and generalized Reynolds numbers are shown in Table 2. 
Table 1 Fanning friction factor equations and application ranges.

\begin{tabular}{|c|c|c|c|c|c|}
\hline No. & Model & Formula & Notes & Year & Ref. \\
\hline 1 & $\begin{array}{l}\text { Dodge \& Metzner } \\
(D \& M)\end{array}$ & $1 / \sqrt{f}=\frac{4}{n^{0.75}}\left(N_{R e g} f^{1-n / 2}\right)-\frac{0.4}{n^{1.2}}$ & $\begin{array}{l}0.36<n<1.0 \\
2,900<N_{\text {Reg }}<100,000\end{array}$ & 1959 & [9] \\
\hline 2 & $\begin{array}{l}\text { Dodge \& } \\
\text { Metzner-Blasius Type } \\
(D \& M)_{B}\end{array}$ & $f=\frac{0.0665+0.01175 n}{N_{R e g}^{0.365-0.177 n+0.0625 n^{2}}}$ & $\begin{array}{l}0.36<n<1.0 \\
2,900<N_{R e g}<100,000\end{array}$ & 1959 & [9] \\
\hline 3 & $\begin{array}{l}\text { Shaver \& Merrill } \\
(\mathrm{S} \& \mathrm{M})\end{array}$ & $f=\frac{0.079}{n^{5} N_{\text {Reg }}^{2.63} / 10.5^{n}}$ & $\begin{array}{l}0.40<n<1.0 \\
4,000<N_{R e g}<100,000\end{array}$ & 1959 & {$[10]$} \\
\hline 4 & $\begin{array}{l}\text { Tomita } \\
\text { (To.) }\end{array}$ & $1 / \sqrt{f}=\sqrt{2} \log \left(N_{R e g} \sqrt{\frac{f}{4}}\right)-\sqrt{0.2}$ & $\begin{array}{l}0.178<n<0.952 \\
2,000<N_{\text {Reg }}<100,000\end{array}$ & 1959 & [17] \\
\hline 5 & $\begin{array}{l}\text { Thomas } \\
\text { (Th.) }\end{array}$ & $1 / \sqrt{f}=\frac{\sqrt{2}}{n} \log \left(N_{R e g}\left(\frac{f}{4}\right)^{1-\frac{n}{2}}\right)-\frac{\sqrt{0.2}}{n}$ & $\begin{array}{l}0.36<n<1.0 \\
2,900<N_{\text {Reg }}<100,000\end{array}$ & 1960 & [18] \\
\hline 6 & $\begin{array}{l}\text { Clapp } \\
\text { (Cl.) }\end{array}$ & $\begin{aligned} 1 / \sqrt{f}=\frac{1.16}{n}-1.22 & +\frac{1.51}{n} \log \left(N_{\text {Reg }}\left(\frac{f}{4}\right)^{1-\frac{n}{2}}\right) \\
+ & \frac{0.58}{n}(5 n-8)\end{aligned}$ & $\begin{array}{l}0.698<n<0.813 \\
548<N_{\text {Reg }}<42,800\end{array}$ & 1961 & [19] \\
\hline 7 & $\begin{array}{l}\text { Kemblowski and } \\
\text { Kolodziejski } \\
(\mathrm{K} \& \mathrm{~K})\end{array}$ & $\begin{array}{c}f=E \times \frac{\emptyset^{\frac{1}{N_{R e g}}}}{N_{R e g}^{\alpha}} \\
E=0.0089 e^{3.57 n^{2}} \\
\emptyset=e^{\frac{0.572\left(1-n^{4.2}\right)}{n^{0.0435}}} \\
\alpha=0.314 n^{2.3}-0.064\end{array}$ & $\begin{array}{l}0.14<n<0.83 \\
2,680<N_{\text {Reg }}<98,600\end{array}$ & 1973 & {$[20]$} \\
\hline 8 & $\begin{array}{l}\text { Hanks \& Ricks } \\
\text { (H \& Ri.) }\end{array}$ & $f=0.0682 \frac{n^{-0.5}}{N_{\text {Reg }}^{\left(\frac{1}{1.87+2.39 n}\right)}}$ & $\begin{array}{l}0.4<n<0.82 \\
4,000<N_{R e g}<75,000\end{array}$ & 1975 & {$[21]$} \\
\hline 9 & $\begin{array}{l}\text { Stein and Kessler } \\
\text { and Greendar } \\
(\mathrm{SKG})\end{array}$ & $1 / \sqrt{f}=1.7373 \ln \left(N_{R e g} f^{0.5}-0.398\right)$ & $\begin{array}{l}0.58<n<0.80 \\
3,400<N_{\text {Reg }}<63,000\end{array}$ & 1980 & {$[22,23]$} \\
\hline 10 & $\begin{array}{l}\text { Szilas, Bobok \& } \\
\text { Navratile } \\
\text { (SBN) }\end{array}$ & $\begin{aligned} 1 / \sqrt{f}=\frac{\sqrt{2}}{n} \log \left(N_{\text {Reg }} f^{1-n / 2}\right) & \\
+ & 1.23^{1 / n}\left(\frac{0.707}{n}+2.12\right) \\
& -\frac{2}{n}-1.028\end{aligned}$ & $\begin{array}{l}0.24<n<1.0 \\
10,000<N_{\text {Reg }}<100,000\end{array}$ & 1981 & [12] \\
\hline 11 & $\begin{array}{l}\text { Garica \& Steffe } \\
(\mathrm{G} \& \mathrm{~S})\end{array}$ & $1 / \sqrt{f}=1.318 \ln \left(N_{R e g} \sqrt{f}-0.398\right)$ & $\begin{array}{l}0.4<n<0.82 \\
3,000<N_{\text {Reg }}<50,000\end{array}$ & 1986 & [24] \\
\hline 12 & $\begin{array}{l}\text { Hartnet \& Rao (H \& } \\
\text { Ra.) }\end{array}$ & $f=0.079 n^{0.675} N_{R e g}^{-0.25}$ & $\begin{array}{l}0.24<n<0.53 \\
3,400<N_{R e g}<11,600\end{array}$ & 1987 & [7] \\
\hline 13 & $\begin{array}{l}\text { Irvine } \\
\text { (Ir.) }\end{array}$ & $f=\frac{2\left(\frac{2^{n}}{7^{7 n}}\right)^{1 /(3 n+1)} 8^{(1-n) /(3 n+1)}}{\left(\frac{3 n+1}{4 n}\right)^{\left(3 n^{2}+2 n\right)} /(3 n+1)} N_{\text {Reg }}^{\frac{-1}{3 n+1}}$ & $\begin{array}{l}0.25<n<0.55 \\
3,400<N_{\text {Reg }}<52,800\end{array}$ & 1988 & {$[25]$} \\
\hline 14 & $\begin{array}{l}\text { Tam \& Tiu } \\
(\mathrm{T} \& \mathrm{~T})\end{array}$ & $f=0.0792\left(\frac{n}{0.25+0.75 n}\right)^{2.5} N_{R e g}^{-0.25}$ & $\begin{array}{l}0.24<n<0.6 \\
3,000<N_{\text {Reg }}<50,000\end{array}$ & 1988 & [26] \\
\hline 15 & $\begin{array}{l}\text { Desouky \& El-Emam } \\
(\mathrm{D} \& \mathrm{E})\end{array}$ & $f=0.71 n^{n}\left(0.0112+N_{R e g}^{-0.3185}\right)$ & $\begin{array}{l}0.241<n<0.893 \\
4,000<N_{R e g}<100,000\end{array}$ & 1990 & [27] \\
\hline
\end{tabular}


Table 1 to be continued

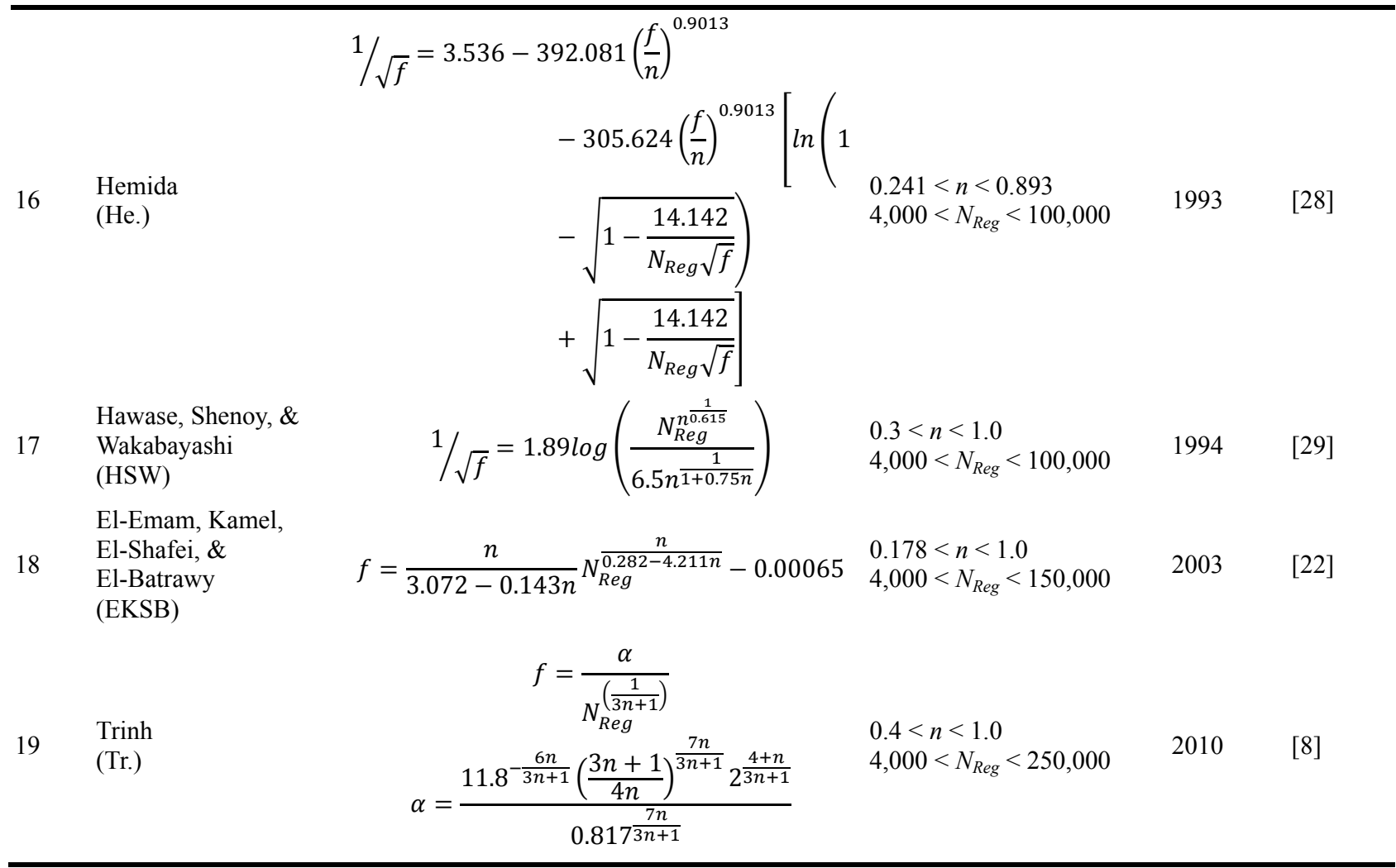

Table 2 Ranges for experimental data.

\begin{tabular}{lllll}
\hline No. & Data & Flow behavior index, $n$ & Generalized Reynolds number, $N_{\text {Reg }}$ & Ref. \\
\hline 1 & Dodge \& Metzner & $0.617-1.0$ & $4,000-100,000$ & {$[9]$} \\
2 & Shaver \& Merrill & $0.6-0.9$ & $5,000-100,000$ & {$[10]$} \\
3 & Bouge & $0.445-1.0$ & $2,100-36,000$ & {$[13]$} \\
4 & Yoo & $0.241-0.893$ & $4,600-57,000$ & {$[11]$} \\
5 & Szilas et al. & $0.5287-0.948$ & $4,000-100,000$ & {$[12]$} \\
6 & Pinho \& Whitelaw & $0.56-0.90$ & $2,000-47,000$ & {$[14]$} \\
7 & Pereira \& Pinho & $0.492-0.685$ & $3,450-100,500$ & {$[15]$} \\
8 & Cruz \& Pinho & $0.40-0.62$ & $2,000-54,000$ & {$[16]$} \\
\hline
\end{tabular}

The different sets of data are considered collectively in the analysis to cover a wide range of both generalized Reynolds number and flow behavior index and to draw a universal conclusion.

\section{Model Selection Criteria}

Various criteria are available for model selection. It is very common that each criterion may lead to a different selection. No definite answer about which criterion should be practiced. Traditionally, the coefficient of determination, $R^{2}$ has been used on a large scale by professional in the oil and gas industry for model selection. However, $R^{2}$ is not the best methods in model selection; alternative approaches are more popular in other fields. Therefore, validity and efficiency is a crucial play when deciding upon which criteria to be used. The six model selection criteria employed in this study are adopted according to strong recommendations [30-36]. This study will detail the six selection criteria and then select the best criterion based on sound mathematical theory. These models under consideration with their details interpretations are listed in Table 3. 
Table 3 Model selection criteria.

\begin{tabular}{|c|c|c|c|}
\hline No. & Criteria & Formula & Notes \\
\hline 1 & $\begin{array}{l}\text { Coefficient of determination, adjusted } \\
\left(\mathrm{R}^{2}\right)\end{array}$ & $R^{2}=1-\frac{\sum_{i=1}^{N}\left(y_{i}-\hat{y}_{i}\right)^{2}}{\sum_{i=1}^{N}\left(y_{i}-\bar{y}_{i}\right)^{2}}$ & $\begin{array}{l}\text { The larger the } R^{2} \text {, the more } \\
\text { accurate the model. }\end{array}$ \\
\hline 2 & $\begin{array}{l}\text { Mean absolute relative error, \% } \\
\text { (MARE) }\end{array}$ & $\begin{array}{c}\text { MARE, } \%=\frac{1}{N} \sum_{i=1}^{n}\left|r_{i}\right| \\
r_{i}=100\left(\frac{\hat{y}_{i}-y_{i}}{y_{i}}\right)\end{array}$ & $\begin{array}{l}\text { The smaller the value, the } \\
\text { better the model. }\end{array}$ \\
\hline 3 & $\begin{array}{l}\text { Root mean square error, \% } \\
\text { (RMSE) }\end{array}$ & $R M S E, \%=\sqrt{\frac{1}{N}} \sum_{i=1}^{N}\left(r_{i}\right)^{2}$ & $\begin{array}{l}\text { The smaller the size of the } \\
\text { errors, the better the } \\
\text { regression model. }\end{array}$ \\
\hline 4 & $\begin{array}{l}\text { Standard deviation of MARE } \\
\left(\mathrm{SD}_{\text {MARE }}\right)\end{array}$ & $\begin{array}{c}S D_{M A R E}=\sqrt{\frac{1}{N} \sum_{i=1}^{N}\left(r_{i}-E_{1}\right)^{2}} \\
E_{1}=\frac{1}{N} \sum_{i=1}^{N} r_{i}\end{array}$ & $\begin{array}{l}\text { Lower values indicate higher } \\
\text { accuracy. }\end{array}$ \\
\hline 5 & $\begin{array}{l}\text { Correlation ratio } \\
(\mathrm{CR})\end{array}$ & $\begin{array}{c}C R=\sqrt{1-\frac{\sum_{i=1}^{N}\left(y_{i}-\hat{y}_{i}\right)^{2}}{\sum_{i=1}^{N}\left(y_{i}-\bar{y}_{i}\right)^{2}}} \\
\bar{y}_{i}=\frac{\sum_{i=1}^{N} y_{i}}{N}\end{array}$ & $\begin{array}{l}\text { Higher values indicate good } \\
\text { agreement. }\end{array}$ \\
\hline 6 & AIC (Akaike information criterion) & $A I C=-2 \log (l(\hat{\theta} \mid y))+2 K$ & $\begin{array}{l}\text { The most appropriate model } \\
\text { is the one with the smallest } \\
\text { values. }\end{array}$ \\
\hline
\end{tabular}

\subsection{The Adjusted Coefficient of Multiple} Determinations, $R^{2}$

The coefficient of multiple determinations measures the proportion of the variation of certain parameter around the mean value. The adjusted coefficient of multiple determination considers the degrees of freedom of the numerator and denominator of $R^{2}$. There are several shortcomings with this criterion. For example, $R^{2}$ is interpreted as an indication of the "goodness of fit" of the model, which may be misleading if data are associated with noise. Another interpretation of the $R^{2}$ coefficient is that the higher the coefficient of determination, the better the variance. Yet, $R^{2}$ can be potentially increased by adding more independent variables to the model that makes it appear to be better while it is not. A third problem is that it does not give a clear cut answer on what value of $R^{2}$ should be used to categorize a good model versus a poor model [1].

\subsection{MARE (Mean Absolute Relative Error)}

It is a relative measure, expressed as percentage, of the amount of physical error when predicting a certain parameter using specific model. It gives an indication of how good the prediction is relative to the measured parameter. Higher errors are more worrying than lower errors. It is simple to calculate and easy to understand. In addition, it is easily and intuitively interpretable, which makes it very popular in our daily language.

However, its validity is questionable. It is neither a resistant nor a robust measure since it can be dominated by the presence of outliers and yields erroneous results. It also lacks the theoretical background [37, 38].

\subsection{RMSE (Root Mean Square Error)}

RMSE (also called the root mean square deviation, $R M S D$ ) is a natural measure used in many forecast 
error evaluations that use regression-based and statistical methods. It is the measure of the noise in the system as it measures the difference between values predicted by a specific model and the observed values. The differences are normally called residuals and can be aggregated into a single measure by the RMSE.

Yet, $R M S E$ is not a good indicator of average model performance and might be misleading as there is no absolute criterion for a "good" fit. Moreover, as arithmetic means, the presence of outliers will influence RMSE [30-38].

\subsection{Standard Deviation of MARE}

It is commonly used and easy to interpret as it gives the standard deviation of the model prediction error. It is a measure of how precise the average is, that is, how well the individual numbers agree with each other. It measures a specific type of error called random error. It is often used to compare real-world data against a model to test it and it has a wide application in all fields [31-36].

\subsection{Correlation Ratio, $C R$}

When the data show a nonlinear relationship between two variables, the correlation ratio is preferred over the coefficient of determination to achieve better answer. It is defined as the ratio of two standard deviations representing variations of dispersion of individual categories over dispersion across the whole sample. It reduces to coefficient for linear relationships. It is used to quantitatively describe the accuracy of model outputs. A correlation ratio of one corresponds to a perfect match between model and observations. Essentially, the closer the value is to one, the more accurate the model is [30-36].

\subsection{AIC}

AIC, in the information theory approach, it is thought of the full reality as a model to be approximated [6] and the objective is to find the model that best approximates the truth model. Akaike showed that the model that best approximates the truth model is the one with smallest value of AIC [39].

The value of AIC gives the information lost if the chosen model is used to approximate the truth model. In other words, in the information theory approach, the smaller the AIC, the more accurate is the model. It is useful to define the AIC difference as $\Delta_{\mathrm{i}}=\mathrm{AIC}_{\mathrm{i}}$ $\mathrm{AIC}_{\text {min }}$, where $\mathrm{AIC}_{\text {min }}$ is the smallest value of the $\mathrm{AIC}$ values for all the set of candidate models. In regard with $\Delta$ values, the best model has a $\Delta$ value of zero. A candidate model with $\Delta$-value higher than 10 should not be considered as a decent model $[3,5]$.

Another parameter is the Akaike's Weight, $w_{\mathrm{i}}$ :

$$
w_{i}=\frac{\exp \left(-\frac{\Delta_{i}}{2}\right)}{\sum_{r=1}^{m} \exp \left(-\frac{\Delta_{i}}{2}\right)}
$$

$\Delta_{i}$ is the AIC difference of the model $i$ and $m$ is the number of candidate models. $w_{i}$ gives the weight of evidence in favor of model $i$ being the best model in the set of models.

The ratio of evidence of a model $g$ is the Akaike weight of the best model divided by the Akaike weight of specific model. It gives an evidence of a kind of weak or strong support for the best model versus any other model in the set of candidate models

One of the approaches to create a $95 \%$ confidence set of models in the information theory approach is based on Akaike weights. In this approach, we sum the Akaike weights from largest to smallest until the sum is just $\geq 0.95$. In the information theory approach, it is essential to find the Akaike weight for each model to be able to see the probability of the model to be the best model. Akaike weight, AIC differences and the confidence set of models are all essential tools in the model selection process in the information theory approach.

While, AIC is theoretically sound and information-based, more stable in model selection and clearly excludes the models that should not be considered, all other methods do not. 


\subsection{Model Analysis; Which Model Is the Best?}

In general, predicted friction factors are compared with the measured ones to evaluate the reliability of a correlation. However, since the selected correlations are either empirical or semi-empirical, and their predictions quite agree with their individual particular experimental data, the comparison was conducted against all the eight sets of experimental data, collectively.

Predictions from the nineteen selected models of friction factor were calculated. Iterations were done to calculate friction factor from the implicit models. The predicted data were compared and evaluated against the eight sets of experimental data, collectively. The evaluation included the six most commonly used statistical parameters and the statistical results are shown in Table 4. Based on the statistical analysis, it can be seen that:

(1) All the models' predictions have $R^{2}$ and $C R$ values higher than 0.25 and 0.45 , respectively.

(2) According to AIC, only one equation, El-Emam et al., shows no information loss $(\Delta=0)$. On the other hand, two equations, Trinh and Hemida equations, show zero weight factor $(w=0)$.

(3) El-Emam et al. and Szilas, et al. equations are more accurate than all other equations considering the entire six criterion. The two equations show the highest $R^{2}$ and $C R$ values and the lowest MARE, RMSE, and $S D_{M A R E}$ values. For AIC, the two equations show very comparable results with very low AIC difference ( 0 and 0.96 , respectively), highest weight factor, $w(0.55$ and $0.34)$ and smallest AIC value (-215.88 and -201.28).

(4) Dodge and Metzner-Blasius type equation comes with an acceptable accuracy level right after the above-mentioned two equations considering most of the model selection criteria.

(5) However, the $R^{2}$ and $C R$ values show other equations (Shaver and Merrill and Stein et al, respectively) to have better accuracy than Dodge and Metzner-Blasius type equation.

(6) Tomita, Hemida, and Trinh equations show the lowest performance compared to other equations considering, at least, four selection criteria, respectively. They show the highest MARE, RMSE, and SDMARE values.

Table 4 Statistical Results.

\begin{tabular}{|c|c|c|c|c|c|c|c|c|c|c|}
\hline \multirow{2}{*}{ No. } & \multirow{2}{*}{ Model } & \multirow{2}{*}{$\left(R^{2}\right)$} & \multirow{2}{*}{$(M A R E)$} & \multirow{2}{*}{$(R M S E)$} & \multirow{2}{*}{$\left(S D_{\text {MARE }}\right)$} & \multirow{2}{*}{$(C R)$} & \multicolumn{4}{|c|}{ (AIC) } \\
\hline & & & & & & & $\Delta$ & $w$ & (AIC) & $g$ \\
\hline 1 & $(\mathrm{D} \& \mathrm{M})$ & 0.72 & 22.59 & 26.26 & 26.07 & 0.89 & 2.95 & 0.21 & -181.52 & 2.6 \\
\hline 2 & $(\mathrm{D} \& \mathrm{M})_{\mathrm{B}}$ & 0.75 & 12.13 & 14.48 & 14.41 & 0.87 & 1.62 & 0.27 & -199.75 & 2.0 \\
\hline 3 & $(\mathrm{~S} \& \mathrm{M})$ & 0.86 & 13.95 & 16.00 & 15.90 & 0.88 & 30.45 & 0.11 & -133.14 & 5 \\
\hline 4 & (To.) & 0.28 & 51.68 & 56.51 & 56.04 & 0.82 & 55.68 & 0.005 & -70.14 & 110 \\
\hline 5 & (Th.) & 0.79 & 15.40 & 18.40 & 18.28 & 0.89 & 5.15 & 0.22 & -185.14 & 2.5 \\
\hline 6 & (Cl.) & 0.78 & 18.73 & 32.63 & 21.84 & 0.78 & 11.25 & 0.21 & -165.92 & 2.6 \\
\hline 7 & $(\mathrm{~K} \& \mathrm{~K})$ & 0.26 & 38.28 & 52.03 & 51.76 & 0.71 & 18.16 & 0.22 & -121.51 & 2.5 \\
\hline 8 & (H \& Ri.) & 0.48 & 14.45 & 15.15 & 15.01 & 0.88 & 25.68 & 0.01 & -135.19 & 55.6 \\
\hline 9 & $(\mathrm{SKG})$ & 0.52 & 43.98 & 49.01 & 48.61 & 0.92 & 31.11 & 0.15 & -118.96 & 3.7 \\
\hline 10 & $(\mathrm{SBN})$ & 0.91 & 11.59 & 14.49 & 14.26 & 0.93 & 0.96 & 0.34 & -201.28 & 1.6 \\
\hline 11 & $(\mathrm{G} \& \mathrm{~S})$ & 0.21 & 22.51 & 45.18 & 50.36 & 0.46 & 36.72 & 0.13 & -145.12 & 4.2 \\
\hline 12 & (H \& Ra.) & 0.53 & 15.76 & 17.20 & 17.05 & 0.90 & 52.52 & 0.09 & -99.14 & 6.3 \\
\hline 13 & (Ir.) & 0.78 & 15.36 & 16.24 & 16.07 & 0.80 & 41.29 & 0.04 & -131.52 & 13.7 \\
\hline 14 & $(\mathrm{~T} \& \mathrm{~T})$ & 0.81 & 16.49 & 17.23 & 17.09 & 0.90 & 2.64 & 0.18 & -177.48 & 3.1 \\
\hline 15 & (D \& E) & 0.78 & 21.50 & 25.63 & 25.45 & 0.91 & 61.55 & 0.01 & -91.82 & 55.6 \\
\hline 16 & (He.) & 0.83 & 52.36 & 77.37 & 77.10 & 0.56 & 70.15 & 0.0 & -65.85 & $\infty$ \\
\hline 17 & (HSW) & 0.69 & 23.73 & 27.36 & 27.16 & 0.83 & 14.58 & 0.24 & -127.77 & 2.3 \\
\hline 18 & (EKSB) & 0.92 & 11.42 & 13.39 & 11.31 & 0.95 & 0.0 & 0.55 & -215.88 & 1 \\
\hline 19 & (Tr.) & 0.49 & 57.45 & 61.12 & 60.57 & 0.86 & 72.84 & 0.0 & -90.36 & $\infty$ \\
\hline
\end{tabular}


(7) For AIC, they exhibit higher AIC difference, lower weight factors and higher AIC values.

(8) Yet, the $R^{2}$ shows a better performance for Hemida equation and assumes Garica and Steffe equation among the worst performer. On the other hand, $C R$ values exclude Trinh equation and replace it by Kemblowski and Kolodziejski equation.

(9) The other 13 equations (excluding the best three performers and the worst three performers listed above) show diverse performance considering the six criteria. Nevertheless, they show poor performance than the best three equations and better performance than the worst three equations.

(10) The results from all the six model selection criteria confirm El-Emam et al. and Szilas, et al. equations as the best models. This is not true for the worst models where only five, out of six, criteria confirm Tomita and Hemida as the worst performer. $C R$ and $R^{2}$ values yield different results.

(11) While $C R$ values list Hemida equation among the worst performer, it shows an acceptable $R^{2}$ values, higher than 0.8 .

(12) The $R^{2}$ and $C R$ values list Kemblowski and Kolodziejski among the worst performer with 0.31 and 0.78 , respectively.

(13) The six-selection criteria agree on selecting the best two models. However, there is no such agreement for the worst two models.

(14) The ratio of evidence, $g$ confirms El-Emam et al. equation as the best model $(g=1)$ and both of Trinh and Hemida as the worst ones $(g=\infty)$.

\section{Results \& Recommendations}

\subsection{Recommended Friction Factor Equation}

According to $A I C$, if $\Delta$ is more than 10 , the model should not be considered. Therefore, there are only 6 models to be considered when predicting friction factor. These models are El-Emam et al., Szilas et al., Dodge and Metzner (both forms), Tam and Tiu, and Thomas equations. Among these six models, Tam and
Tiu and Thomas show weak performance considering other selection criteria when compared to top four equations. Therefore, the top four models are the only recommended ones, from the authors' point of view considering all the statistical results.

Among these four equations, the statistical results demonstrate clearly that the El-Emam et al. and Szilas et al. models are the best two models that show excellent agreement with the measured data considering the six selection criteria over the complete range of $n$ and $N_{\text {Reg }}$ values. Dodge and Metzner correlation (both forms) can, therefore, be excluded as they show relatively lower $R^{2}$ (did not exceed 0.75 ) and low $C R$ (did not exceed 0.89 ) values compared to El-Emam et al. and Szilas et al. models.

Yet, El-Emam et al. model has no information loss $(\Delta=0)$ while Szilas et al. model shows an information loss of 0.96. Meanwhile, El-Emam et al. model has the largest Akaike weight factor of $(w=0.55)$, while it is relatively low for Szilas et al. model $(w=0.34)$.

A value of 0.55 , the weight of evidence, is in favor of El-Emam et al. equation being the best in the set of all equations. It shows a much better performance compared to Szilas et al. equation as the ratio between their Akaike weights (ratio of evidence, $g$ is $0.55 / 0.34$ $=1.6$ ) which states that El-Emam et al. equation is 1.6 times better than the other one.

In addition, El-Emam et al. equation covers a wider range of flow behavior indices $(0.178<n<1.0)$ and Reynolds number $\left(4,000<N_{\text {Reg }}<150,000\right)$ than what Szilas et al. equation covers $(0.24<n<1.0$ and 4,000 $\left.<N_{\text {Reg }}<150,000\right)$. Moreover, it has another advantage because of its simplicity in calculation and explicit form. Consequently, it is strongly recommended by the authors for friction factor prediction in the future.

This analysis clearly illustrates that some of the very common selection criteria can be misleading. The $R^{2}$ values for five equations are more than 0.8 indicating relatively reasonable similarity between predicted and measured friction factors. On the other 
hand, the $C R$ values for fourteen equations are more than 0.8 (six of them show a value higher than 0.9) indicating their high performance. Yet, these models should not be considered when predicting friction factor as they show a $\Delta$-value higher than 10 according to AIC. Furthermore, considering MARE, $R M S E$, and $S D_{M A R E}$, these equations do not exhibit the same performance as indicated by either $R^{2}$ or $C R$.

Considering all criteria, it can be seen that, every selection criterion shows somehow a different order for the nineteen friction factor equations investigated in this study as shown in Table 5. Yet, all the six criteria agree when selecting the top two models, which is not the case when talking about the worst models. Furthermore, there is no clear answer when using some selection criteria, high $R^{2}$, high, $C R$, etc. On the contrary, it is clear that the AIC criteria confirm largely the models that exhibit the best performance and rank them according to the information loss and their weight factor. El-Emam et al. equation has an accuracy 1.6 times $(g=0.55 / 0.34=$ 1.6) better than Szilas et al. model (the second best model) and more than two times $(\mathrm{g}=0.55 / 0.27=2)$ better performance than Dodge and Metzner-Blasius type model. Similar conclusions can be drawn from the other four selection criteria, $R^{2}, M A R E, S D_{M A R E}$, and $C R$. The RMSE values are slightly different as its value ranks Dodge and Metzner-Blasius type model as the second best model favoring it over Szilas et al. model, which comes in the third rank. However, the difference is not significant.

Since the main goal is to select the best model among different models available to predict certain parameter, giving no weight to the worst models, any selection criteria can be used. Favorably, the AIC criterion provides greater insight on the validity of each equation. In particular, AIC ranking clearly indicates not the only the best equation and how it is compared to the other ones, but also it ranks the equation and separates the ones that should not be considered. Its efficiency is clear and it shows how and why the selected equation is preferred. The advantages of using AIC criterion over the other are explained in more details in other work $[1,40,41]$.

Table 5 Order of the friction factor equations.

\begin{tabular}{|c|c|c|c|c|c|c|c|}
\hline No. & Model & $\left(R^{2}\right)$ & (MARE) & (RMSE) & $\left(S D_{\text {MARE }}\right)$ & $(C R)$ & (AIC) \\
\hline 1 & $(\mathrm{D} \& \mathrm{M})$ & 11 & 13 & 11 & 12 & 7 & 5 \\
\hline 2 & $(\mathrm{D} \& \mathrm{M})_{\mathrm{B}}$ & 10 & 3 & 2 & 3 & 11 & 3 \\
\hline 3 & $(\mathrm{~S} \& \mathrm{M})$ & 3 & 4 & 5 & 5 & 9 & 10 \\
\hline 4 & (To.) & 17 & 17 & 17 & 17 & 14 & 19 \\
\hline 5 & (Th.) & 6 & 7 & 9 & 9 & 8 & 4 \\
\hline 6 & (Cl.) & 7 & 10 & 13 & 10 & 16 & 7 \\
\hline 7 & $(\mathrm{~K} \& \mathrm{~K})$ & 18 & 15 & 16 & 16 & 17 & 13 \\
\hline 8 & (H \& Ri.) & 16 & 5 & 4 & 4 & 10 & 9 \\
\hline 9 & $(\mathrm{SKG})$ & 14 & 16 & 15 & 14 & 3 & 14 \\
\hline 10 & $(\mathrm{SBN})$ & 2 & 2 & 3 & 2 & 2 & 2 \\
\hline 11 & $(\mathrm{G} \& \mathrm{~S})$ & 19 & 12 & 14 & 15 & 19 & 8 \\
\hline 12 & (H \& Ra.) & 13 & 8 & 7 & 7 & 5 & 15 \\
\hline 13 & (Ir.) & 8 & 6 & 6 & 6 & 15 & 11 \\
\hline 14 & $(\mathrm{~T} \& \mathrm{~T})$ & 5 & 9 & 8 & 8 & 6 & 6 \\
\hline 15 & $(\mathrm{D} \& \mathrm{E})$ & 9 & 11 & 10 & 11 & 4 & 16 \\
\hline 16 & (He.) & 4 & 18 & 19 & 19 & 18 & 8 \\
\hline 17 & (HSW) & 12 & 14 & 12 & 13 & 13 & 12 \\
\hline 18 & (EKSB) & 1 & 1 & 1 & 1 & 1 & 1 \\
\hline 19 & (Tr.) & 15 & 19 & 18 & 18 & 12 & 17 \\
\hline
\end{tabular}




\section{Summary}

This paper presents a new statistical evaluation to examine 19 friction factor correlations for a flow behavior index, $n$ from 0.14 to 1.0 and $N_{\text {Reg }}$ from 548 to 250,000 . Six different model selection criteria and eight different sets of experimentally measured friction factor were used. The following are the concluded remarks:

(1) El-Emam et al. model is strongly recommended for predicting friction factors under turbulent flow conditions.

(2) Other models with a relatively lower confidence level such as Szilas et al. and Dodge and Metzner models can be recommended.

(3) Different selection criteria result in different ranking of the investigated correlations. Yet, nearly all criteria recommended the best model and the worst model.

(4) The use of the $R^{2}$ and $C R$ is inappropriate, especially when selecting the worst model.

(5) MARE, RMSE, and SD MARE can be used to rank models, but do not give in-depth details about the validity and ranking.

(6) AIC is a new tool that can best help to select the accurate models and advances the state-of-the-art of models comparison. AIC is theoretically sound, more stable and shows numerous advantages over the other presented criteria.

(7) Future investigation is proposed using other friction factor correlations and other model selection criteria for better understanding of the model selection challenge.

\section{References}

[1] Kamel, A. H., Shaqliah, A. S., and Ibrahim, E. 2015. "Model Inference using the Akaike Information Criterion for Turbulent Flow of non-Newtonian Crude Oils in Pipelines." Petroleum Science 12: 492-500. doi: 10.1007/s12182-015-0032-y.

[2] Rudman, M., Blackburn, H. M., Graham, L., and Pullum, L. 2004. "Turbulent Pipe Flow of Shear-Thinning Fluids." J. Non-Newtonian Fluid Mech. 118: 33-48.
[3] Anderson, D. 2008. Model Based Inference in the Life Sciences: A Primer on Evidence. New York, NY: Springer.

[4] Shaqlaih, A. 2010. "Model Selection by an Information Theory Approach." Ph.D. thesis, University of Oklahoma, Norman, OK, USA.

[5] Shaqlaih, A., White, L., and Zaman, M. 2013. "Resilient Modulus Modeling with Information Theory Approach." International Journal of Geomechanics 13 (4): 384-9.

[6] Burnham, D., and Anderson, D. 2002. Model Selection and Multimode Inference: A Practical Information-Theoretic Approach (2nd ed.). New York, NY: Springer.

[7] Hartnet, J. P., and Kostic, M. 1990. "Turbulent Friction Factor Correlations for Power Law Fluids in Circular and non-Circular Channels." Int. Com. Heat Mass Trans. 17: 59-65.

[8] Trinh, K. 2010. "A General Correlation for Turbulent Friction Factors in Non-Newtonian Fluids." In Proc. 7th World Congress of Chemical Engineering, Glasgow, UK. Rugby, UK: The Institution of Chemical Engineers. arXiv:1009.0968.

[9] Dodge, D., and Metzner, A. 1959. "Turbulent Flow of Non-Newtonian Systems." AIChE Journal 5 (2): 189-203.

[10] Shaver, R., and Merrill, E. 1959. "Turbulent Flow of Pseudoplastic Polymer Solutions in Straight Cylindrical Tubes." AIChE Journal 5 (2): 181-8.

[11] Yoo, S. S. 1974. "Heat Transfer and Friction Factors for non-Newtonian Fluids in Turbulent Pipe Flow." Ph.D. thesis, Graduate College, University of Illinois, Chicago, USA.

[12] Szilas, A., Bobok, E., and Navratil, L. 1981. "Determination of Turbulent Pressure Losses of Non-Newtonian Oil Flow in Rough Pipes." Rheological Acta 20 (5): 487-96.

[13] Bogue, D. C. 1962. "Velocity Profiles in Turbulent Non-Newtonian Pipe Flow." Ph.D. Thesis, University of Delaware, Newark, Delaware, U.S.

[14] Pinho, F. T., and Whitelaw, J. H. 1990. "Flow of Non-Newtonian Fluids in a Pipe." Journal of Non-Newtonian Fluid Mechanics 34 (2): 129-44.

[15] Pereira, A. S., and Pinho, F. T. 2002. "Turbulent Pipe Flow of Thixotropic Fluids." International Journal of Heat and Fluid Flow 23 (1): 36-51.

[16] Cruz, D. O., and Pinho, F. T. 2003. "Turbulent Pipe Flow Predictions with a Low Reynolds Number $k-\varepsilon$ Model for Drag Reducing Fluids." Journal of Non-Newtonian Fluid Mechanics 114 (2-3): 109-48.

[17] Tomita, Y. 1959. "A Study of Non-Newtonian Flow in Pipelines.” Bulletin JSME 2: 10-6. 
[18] Thomas, A. 1960. "Heat and Momentum Transport Characteristics of Non-Newtonian Aqueous Thorium Oxide Suspensions." AIChE Journal 6: 631-9.

[19] Clapp, R. 1961. "Turbulent Heat Transfer in Pseudoplastic Non-Newtonian Fluids." Int. Developments in Heat Transfer, ASME, Part III, Sec. A, 652-61.

[20] Kemblowski, Z., and Kolodzie, J. 1973. "Flow Resistances of Non-Newtonian Fluids in Transitional and Turbulent-Flow." International Chemical Engineering 13 (2): 265-79.

[21] Hanks, R., and Ricks, B. 1975. "Transitional and Turbulent Pipe Flow of Pseudoplastic Fluids.” Journal of Hydronautics 9: 39-44.

[22] El-Emam, N., Kamel, A., Al-Shafie, M., and Al-Batrawi, A. 2003. "New Equation Calculates Friction Factor for Turbulent Flow of Non-Newtonian Fluids." Oil \& Gas Journal 101 (36): 74-83.

[23] Gao, P., and Zhang, J. 2007. "New Assessment of Friction Factor Correlations for Power Law Fluids in Turbulent Pipe Flow: A Statistical Approach.” J. Cent. South Univ. Technol. 14 (s1): 0077-815. doi: 10.1007/s11771-007-0219-5.

[24] Garica, E. J., and Steffe, J. F. 1987. "Comparison of the Friction Factor Equations for Non-Newtonian Fluids in Pipe Flow." Journal of Food Process Engineering 9 (2): 93-120.

[25] Irvine, Jr., T. F., 1988. "Friction Factor for Power Las Fluids." Chem. Eng. Commun. 65: 39.

[26] Tam, K. C., and Tiu, C. 1988. "A General Correlation for Purely Viscous Non-Newtonian Fluids Flowing in Ducts of Arbitrary Cross Sections." The Canadian Journal of Chemical Engineering 66: 542-9.

[27] Desouky, S., and El-Emam, N. 1990. "A Generalized Pipeline Design Correlation for Pseudoplastic Fluids." Journal of Canadian Petroleum Technology 29 (5): 48-54.

[28] Hemeida, A. M. 1993 "Friction Factor for Yieldless Fluids in Turbulent Pipe Flow." Journal of Canadian Petroleum Technology 32 (1): 32-5.

[29] Hawase, Y., Shenoy, A., and Wakabayashi, K. 1994. "Friction and Heat and Mass Transfer for Turbulent Pseudoplastic Non-Newtonian Fluids Flowing in Rough Pipes." The Canadian Journal of Chemical Engineering 72: 798-804.

[30] Gregory, G. A., and Fogarasi, M. 1985. "Alternate to Standard Friction Factor Equation." Oil Gas Journal 83 (13): $120-7$.
[31] Xiao, J. J., Shonham, O., and Brill, J. P. 1990. "A Comprehensive Mechanistic Model for Two-Phase Flow in Pipelines." Presented at SPE 20631, the 65th SPE Annual Technical Conference and Exhibition, New Orleans, LA.

[32] Ouyang, L. 1998. "Single Phase and Multiphase Fluid Flow in Horizontal Wells." Ph.D. Dissertation Thesis. Dept. of Petro. Eng., School of Earth Sciences. Stanford University.

[33] García, F., García, R., Padrino, J. C., Mata, C., Trallero, J., and Joseph, D. 2003. "Power Law and Composite Power Law Friction Factor Correlations for Laminar and Turbulent Gas-Liquid Flow in Horizontal Pipelines." Int. J. Multiphase Flow 29 (10): 1605-24.

[34] García, F., García, R., and Joseph, D. 2005 "Composite Power Law Hold up Correlations in Horizontal Pipes." Int. J. Multiphase Flow 31 (12): 1276-303.

[35] García, F., García, J. M., García, R., and Joseph, D. 2007. "Friction Factor Improved Correlations for Laminar and Turbulent Gas-Liquid Flow in Horizontal Pipelines." Int. J. Multiphase Flow 33: 1320-36.

[36] Genić, S., Arandjelović, I., Kolendić, P., Jarić, M., Budimir, N., and Genić, V. 2011. "A Review of Explicit Approximations of Colebrook's Equation." FME Transactions 39: 67-71.

[37] Tofallis, C. 2015. "A Better Measure of Relative Prediction Accuracy for Model Selection and Model Estimation." Journal of the Operational Research Society 66 (8): 1352-62.

[38] Chail, T., and Draxler, R. 2014, "Root Mean Square Error (RMSE) or Mean Absolute Error (MAE)?-Arguments Against Avoiding RMSE in the Literature." Geosci. Model Dev. 7: 1247-50. doi:10.5194/gmd-7-1247-2014.

[39] Akaike, H. 1973. "Information Theory as an Extension of the Maximum Likelihood Principal." In Proceedings of the 2nd International Symposium on Information Theory Budapest, 267-81.

[40] Shaqlaih, A. S., and Kamel, A. H. 2013. "AIC Applications in Coiled Tubing Hydraulics." International Journal of Petroleum \& Geoscience Engineering 1 (2): 62-81.

[41] Shaqlaih, A. S., and Kamel, A. H. 2014. "A Statistical Evaluation of the Frictional Pressure Losses Correlations for Slurry Flow." Journal of Geological Resource and Engineering 2 (2): 69-76. doi: $10.17265 / 2328-2193 / 2014.02 .001$. 